\section{Water Hardness in the Eye of the Beholder: Exploring Links between Central Softening, Customer Perception and Behavior, and Citizen Science}

\section{STIJN BROUWER (D)}

\section{DIMITRIOS BOUZIOTAS (D)}

*Author affiliations can be found in the back matter of this article

\section{ABSTRACT}

Despite the significant growth of citizen science (CS) initiatives in number, scale, and scope, few studies have taken an empirical approach to studying the outcomes for individual participants. This paper analyzes these outcomes within the drinking water domain and assesses to what extent, if at all, CS participation leads to more profound perception and behavior change as compared with non-CS participation, i.e., the control group. Our empirical research is based around a CS project studying the hardness and lime deposition of drinking water in the Flemish Waarmaarde area, where, during the project period, water hardness was lowered through the installation of a central softener. The CS participants performed two hardness measurement rounds, both prior and following the softener installation. To monitor the difference in perception and behavior between CS participants and non-CS participants, both before and after this intervention, complementary quantitative surveys were conducted at three different stages (baseline and pre- and post-softener installation). The results showed that the central softening of water can lead to significant positive shifts in the perception of, among other factors, hardness, quality, and confidence in the water utility. For several factors, this shift is more profound among CS participants compared with the control group. The same pattern is observed when examining behavior, evidenced for instance in the frequency of use of calcium-removing products.
CORRESPONDING AUTHOR:

\section{Stijn Brouwer}

KWR Water Research Institute, $\mathrm{NL}$

stijn.brouwer@kwrwater.nl

KEYWORDS:

citizen science; tap water quality; perceptions; water hardness; behavior change

TO CITE THIS ARTICLE: Brouwer, $S$ and Bouziotas, D. 2022. Water Hardness in the Eye of the Beholder: Exploring Links between Central Softening, Customer Perception and Behavior, and Citizen Science. Citizen Science: Theory and Practice, 7(1): 2, pp. 1-14. DOI: https://doi.org/10.5334/ cstp.452 


\section{INTRODUCTION}

The steady stream of publications, including three recent literature reviews on crowdsourced data collection within the context of hydrology (Njue et al. 2019), on water quality monitoring (Capdevila et al. 2020), and on citizen science (CS) applications in the water sciences (Walker, Smigaj, and Tani 2021), leads to the conclusion that CS is of increasing importance in the water domain. CS application is growing particularly rapidly in the fields of surface water quantity and quality, soil moisture, mapping exercises, precipitation, and flood risk management (e.g., Ferri et al. 2020; Pudifoot et al. 2021; Topping and Kolok 2021). However, the involvement of non-scientists in the context of drinking water remains, at present, relatively scarce. Examples of efforts in this field include several studies on lead concentrations in drinking water (Jakositz et al. 2020; Redmont et al. 2020), water hardness, and other chemical water quality parameters (Brouwer and Hessels 2019; Babich et al. 2021; D’Alessio et al. 2021; Odetola et al. 2021), as well as studies initiated in the context of the Flint water crisis (e.g., Roy and Edwards 2019) and a study on the microbiological stability of drinking water (Brouwer et al. 2018).

\section{CITIZEN SCIENCE OUTCOMES}

The outcomes of CS projects have been categorized by Shirk et al. (2012) into outcomes for research (e.g., scientific findings), outcomes for the system (e.g., influencing policies), and outcomes for individual participants. The latter outcomes, among others, may relate to the development of new skills, to increasing scientific literacy, to empowerment, and to changing attitudes and behavior (Land-Zandstra, Agnello, and Gültekin 2021; Haywood 2016; Stepenuck and Green 2015; Brouwer and Hessels 2019).

While scientific benefits of CS to researchers are well reported, system and individual outcomes, including behavior change, are frequently overlooked (Jakositz et al. 2020; Jordan et al. 2011). Indeed, based on their extensive literature review, Walker, Smigaj, and Tani (2021) conclude that about one quarter of all water CS papers reviewed had no mention of participant or community benefits, whereas about a third only list benefits without explanation or investigation. The numbers of CS studies that report behavior change is even more limited, with a few notable exceptions such as those that observe enhanced water conservation efforts (Egerer, Lin, and Philpott 2018; Harriden 2013), and those that observe the purchase of rain barrels and ecofriendly cleaning products (Church et al. 2019).

To address this gap, this paper explores how perceptions and behavior change after a particular intervention in the provision of drinking water, i.e., the installation of a central softener, and how, if at all, the impact of this intervention differs between water customers directly involved in a connected CS project, i.e., the group of citizen scientists, and those not involved in this project, i.e., the control group. As further explained below, in this project, CS participants conducted hardness and calcium precipitation measurements, both before and after the installation of the central softener. Although (i) a standard, and therefore identical, drinking water company letter was sent to both groups informing them about the softening intervention, and more importantly, (ii) both groups received tap water of an identical quality both before and after the installation of the central softener, we nevertheless expected that drinking water customers who had the opportunity to personally verify and observe these changes through accurate CS measurements would be more likely to react profoundly to those changes, compared with customers passively experiencing this change. Accordingly, and in line with previous work that found that CS can lead to increased awareness and behavior change (Brouwer and Hessels 2019; Church et al. 2019), our central hypothesis was that after the softening intervention, CS participants would show more profound changes in their quality perceptions and behavior.

\section{HARDNESS OF DRINKING WATER}

While safe drinking water provision still remains a pressing issue in many parts of the world (Li and Wu 2019), modern supply companies also focus on customer comfort, which can be significantly improved by the removal of hardness (Groenendijk, Van de Wetering, and Van Nieuwenhuijze 2008). Water hardness is generally defined as the amount of dissolved minerals in water, mainly calcium and magnesium compounds (Ahn et al. 2018). Hard water utilization in the home not only interferes with laundering, as clothes laundered in hard water may look dingy and harsh, but also with washing, bathing, and personal grooming. Furthermore, it affects soap and detergent use, and may lead to the formation of solid deposits in domestic waterusing appliances and kitchenware. For this reason, hard water can damage and significantly reduce the lifetime of appliances, implying inconvenience and expenses to households (Lanz and Provins 2016; Ahn et al. 2018).

The hardness of water can be mitigated not only at the individual household level by purchasing individual water softener systems, but also by central water company investments in treatment plants (Lanz and Provins 2016). Central softening is the drinking water treatment technology that aims at reducing the calcium/magnesium deposits and corresponding carbonate scaling clients 
experience at the tap level. Besides improving customer satisfaction, central softening leads to cost reductions, a smaller energy footprint during water use, and benefits to the environment (Groenendijk, Van de Wetering, and Van Nieuwenhuijze 2008).

In this study, we differentiate between (i) the general water quality parameter total hardness of water (TH), defined as the sum of calcium and magnesium deposits, and (ii) the practical calcium carbonate precipitation (PCCP), which is a more accurate descriptor of the calcium carbonate precipitation and dissolution (Groenendijk, Van de Wetering, and Van Nieuwenhuijze 2008). The combination of the TH and the PCCP determines customer comfort. The $\mathrm{TH}$ is usually expressed as concentration using molecular weights (e.g., $\mathrm{mol} / \mathrm{m}^{3} \mathrm{CaCO}_{3}$ ) and is, in practice, measured in degrees of German, English, or French hardness. In this study, as commonly practiced in Belgium, we refer to water hardness measurements in their original values using the French system of degrees $\left({ }^{\circ} \mathrm{fH}\right)$, which, as detailed in Table 1, can be readily classified as very soft, soft, average, hard, and very hard water.

We observed, as have others, a strong correlation between the perception of tap water quality and the perception of hardness (Brouwer and Sjerps 2018). People who perceive their water as hard provide consistently lower scores in terms of water quality, and vice versa. In addition, lime deposits directly impact everyday cleaning. And, in an evaluation of previous drinking water CS projects, the topic we present here was the number one idea identified by participants for future study (Brouwer and Hellels 2019). As such, we believe that our case study, to some extent, mirrors Irwin's (1995) original conceptualization of CS. According to Irwin, CS not only implies a form of science enacted by citizens, that is, "science by the people," but also is a form of science that assists their needs and concerns, that is, "science for the people."

\section{METHODS}

Here we present a case study of a CS project in the supply area of the Waarmaarde water production site, which is located in the Province of West-Flanders, Belgium. This Waarmaarde production site is one of the ninety production sites of De Watergroep, the largest drinking water utility in Flanders, and provides water to 13,000 households. During the study, a new water softener installation was introduced at the production site. After installation, customers received water with a hardness of $19.9^{\circ} \mathrm{fH}$, instead of $41.75^{\circ} \mathrm{fH}$, as measured at the production site. To monitor the difference in perception and behavior between CS participants and non-CS participants, both before and after the installation of the central softener, the CS project was accompanied by three complementary quantitative surveys. Figure 1 illustrates the study design and the timeline of activities.

The first survey $(N=827)$, hereafter referred to as the baseline survey, was conducted in 2018 and covered the entire service area of De Watergroep. This survey, which largely mirrored a customer perception survey conducted in the Netherlands as reported by Brouwer et al. (2019), was primarily aimed at gaining a deeper insight into the satisfaction, interests, and concerns of Flemish drinking water customers. The second survey, hereafter referred to as the pre-survey, was aimed at customers living close to the Waarmaarde water production site, that is, the CS pilot study area. The main purpose of this pre-survey (2019) was to analyze the perception of hardness of water prior to the central softening, as well as to get a better understanding of the behavior of customers in relation to hardness and lime deposition, including the frequency of descaling. Presurvey respondents were recruited via e-mail, using the utility client database, and by posting an online survey link on Facebook, published within the study area. After filling out the pre-survey, 152 respondents participated in the CS study. Another 543 respondents either did not give the researchers permission to contact them again for follow-up study and therefore could not be invited for CS participation, or did not accept the invitation for CS participation. As a result, we can, in effect, distinguish between two separate groups: the CS participants ( $\mathrm{n}=$ 152 ) and the control group $(n=543)$. The third survey, hereafter referred to as the post-survey, conducted in December 2020, largely mirrored the pre-survey, in terms of the target respondents, with a CS group $(n=71)$ and a

\begin{tabular}{llll}
\hline HARDNESS IN FRENCH DEGREES ( $\left.{ }^{\circ} \mathrm{FH}\right)$ & PRACTICAL CALCIUM CARBONATE PRECIPITATION (PCCP) \\
\hline$<7^{\circ} \mathrm{fH}$ & Very soft water & (Virtually) no problems with calcium precipitation & $\mathrm{PCCP}<0.3 \mathrm{~mol} / \mathrm{m}^{3} \mathrm{Ca}$ \\
\hline $7-15^{\circ} \mathrm{fH}$ & Soft water & Slight problems with calcium precipitation & $0.3<\mathrm{PCCP}<0.6 \mathrm{~mol} / \mathrm{m}^{3} \mathrm{Ca}$ \\
\hline $15-30^{\circ} \mathrm{fH}$ & Average hard water & Problems with calcium precipitation & $0.6<\mathrm{PCCP}<1.0 \mathrm{~mol} / \mathrm{m}^{3} \mathrm{Ca}$ \\
\hline $30-45^{\circ} \mathrm{fH}$ & Hard water & Serious problems with calcium precipitation & $\mathrm{PCCP}>1.0 \mathrm{~mol} / \mathrm{m}^{3} \mathrm{Ca}$ \\
\hline$>45^{\circ} \mathrm{fH}$ & Very hard water & & \\
\hline
\end{tabular}

Table 1 Classification of hardness and PCCP. 


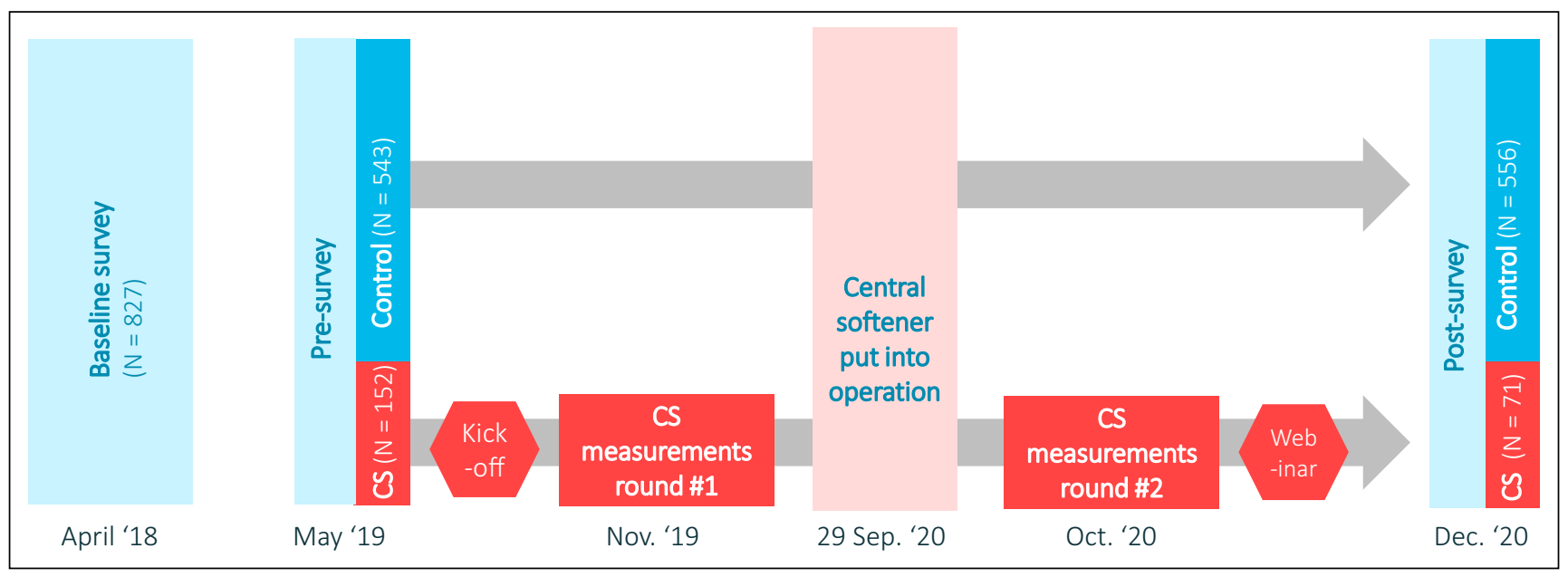

Figure 1 Study design and timeline of activities. All steps followed by the citizen science participants are marked in red. CS: citizen science.

\begin{tabular}{|c|c|c|c|c|c|c|}
\hline & & $\begin{array}{l}\text { BASELINE } \\
\text { SURVEY }\end{array}$ & $\begin{array}{l}\text { PRE-SURVEY } \\
\text { CITIZEN } \\
\text { SCIENCE }\end{array}$ & $\begin{array}{l}\text { PRE-SURVEY } \\
\text { CONTROL }\end{array}$ & $\begin{array}{l}\text { POST-SURVEY } \\
\text { CITIZEN } \\
\text { SCIENCE }\end{array}$ & $\begin{array}{l}\text { POST-SURVEY } \\
\text { CONTROL }\end{array}$ \\
\hline & YEAR & 2018 & 2019 & 2019 & 2020 & 2020 \\
\hline & Number of participants $N$ & 827 & 152 & 543 & 71 & 556 \\
\hline \multirow[t]{6}{*}{ Age } & 24 or younger & $10.6 \%$ & $3.9 \%$ & $1.3 \%$ & $6.3 \%$ & $0.2 \%$ \\
\hline & $25-34$ & $14.0 \%$ & $15.1 \%$ & $22.4 \%$ & $30.2 \%$ & $9.5 \%$ \\
\hline & $35-44$ & $13.7 \%$ & $27.6 \%$ & $24.8 \%$ & $11.1 \%$ & $10.4 \%$ \\
\hline & $45-54$ & $20.3 \%$ & $19.7 \%$ & $19.0 \%$ & $22.2 \%$ & $15.6 \%$ \\
\hline & $55-64$ & $18.7 \%$ & $20.4 \%$ & $15.9 \%$ & $23.8 \%$ & $27.5 \%$ \\
\hline & 65 or older & $22.6 \%$ & $9.9 \%$ & $12.8 \%$ & $6.3 \%$ & $29.7 \%$ \\
\hline \multirow[t]{2}{*}{ Sex } & Male & $49.0 \%$ & $66.4 \%$ & $56.4 \%$ & $71.4 \%$ & $64.8 \%$ \\
\hline & Female & $50.9 \%$ & $33.6 \%$ & $43.6 \%$ & $28.6 \%$ & $35.2 \%$ \\
\hline \multirow[t]{4}{*}{ Education } & Primary school & - & $2.6 \%$ & $4.9 \%$ & $3.2 \%$ & $4.6 \%$ \\
\hline & Secondary education & $65.3 \%$ & $26.3 \%$ & $43.6 \%$ & $38.1 \%$ & $44.5 \%$ \\
\hline & Professional bachelor's degree & $23.7 \%$ & $41.4 \%$ & $31.8 \%$ & $34.9 \%$ & $32.8 \%$ \\
\hline & Master degree & $11.0 \%$ & $29.6 \%$ & $19.7 \%$ & $23.8 \%$ & $18.1 \%$ \\
\hline \multirow[t]{4}{*}{ Perspective } & Aware and committed & $21.9 \%$ & $37.5 \%$ & $23.5 \%$ & $40.3 \%$ & $26.6 \%$ \\
\hline & Quality and health concerned & $21.2 \%$ & $24.3 \%$ & $31.1 \%$ & $11.9 \%$ & $21.6 \%$ \\
\hline & Egalitarian and solidary & $36.6 \%$ & $27.6 \%$ & $25.8 \%$ & $32.8 \%$ & $29.9 \%$ \\
\hline & Down-to-earth and confident & $20.3 \%$ & $10.5 \%$ & $19.5 \%$ & $14.9 \%$ & $21.8 \%$ \\
\hline
\end{tabular}

Table 2 Study sample characteristics.

control group ( $n=556$ ), as well as in terms of questions. The questions of the pre- and post-survey are provided in Supplemental File 1: Survey Questions. The protocol and ethics of this study were reviewed and approved by the Chairman of the Scientific Council of KWR Water Research Institute. In accordance with this protocol, all survey respondents signed electronic informed consent prior to beginning the study.

The characteristics of the study sample for each survey are presented in Table 2. As shown in this table, in this study, both personality traits (such as gender, educational background, and age) and the respondents' subjective 
views on drinking water are considered. For the respondents' views, we built on the work of Brouwer et al. (2019), who distinguish four different customer perspectives on drinking water: (i) the "aware and committed" perspective, characterized by pro-environmental values and collective sustainability ideals; (ii) the "quality and health concerned" perspective, characterized by a focus on personal preferences and needs, especially regarding their own health and tap water quality; (iii) the "egalitarian and solidary" perspective, marked by a great sense of solidarity with less-favored households, developing countries, and future generations; and (iv) the "down-to-earth and confident" perspective, characterized by great confidence in the responsibility of water utilities, along with a desire not to be bothered about drinking water.

As mentioned, the key aim of this study is to examine to what extent perceptions and behavior change after the installation of a central softener, and how, if at all, the impact of this intervention differs between CS participants and non-CS participants. More specifically, we investigated whether changes in perception and behavior are more profound (i.e., statistically different) among the CS participants as a result of their active involvement and measurement effort. To evaluate this argument, we statistically tested a number of hypotheses. Of all possible combinations, the following four are of interest:

- Differences between the pre-survey and post-survey CS datasets, indicating that there is a significant change before and after the installation of the softener in the smaller pool of CS respondents.

- Differences between the pre-survey and post-survey control datasets, indicating that there is a significant change before and after the installation of the softener in the large control pool of respondents.

- Differences between the pre-survey CS and pre-survey control datasets, indicating that there is a significant difference between the CS and control datasets in the pre phase.

- Likewise, differences between the post-survey CS and post-survey control datasets, indicating that there is a significant difference between the CS and control datasets in the post phase.

The survey data were found to be non-normally distributed and with unequal variances at different phases (with the Shapiro-Wilk's and Levene's tests failing for most questions). As a result, non-parametric methods were used to test against the statistical significance of differences (Wilcoxon-Mann-Whitney tests and chi-squared tests, depending on whether the variable is of nominal or ordinal nature).

\section{RECRUITMENT}

In this project, following the classification of Brouwer and Hessels (2019), a combination of a targeted recruitment strategy and a generic recruitment strategy was used. First, targeted e-mail invitations were sent to the pool of respondents ( $n=312$ ) that: (i) participated in the original pre-survey; (ii) indicated no objection to receiving a related follow-up survey: and (iii) resided within the pilot study area. In total, 62 citizens replied with a positive response to this invitation, giving a $19.9 \%$ response rate. Additionally, targeted e-mail invitations were sent to a random sample ( $n=1000)$ of households within the pilot study area. For this group, the positive response rate was merely $2.4 \%$, equaling 24 people. In addition to this targeted invitation strategy, participants were recruited via Facebook-targeted advertising. Within a timespan of two weeks, 66 citizens registered via this Facebook campaign, amounting to a total of 152 registrations.

As depicted in Table 2, about half of the registered volunteering citizen scientists were younger than 45 (48\%); the age group with the most respondents was 35-44 (28\%). The majority of respondents were male (69\% versus $31 \%)$. This figure may partly relate to the fact that the e-mails (target invitation strategy) were sent to the drinking water company's contact persons, that is, persons in charge of paying the bill, which may be more often men. This, however, cannot be the full explanation since men, although less significantly, are also overrepresented in the Facebook campaign, where such bias doesn't play a role. The majority of the respondents were well educated: $41 \%$ with a bachelor's degree and $30 \%$ with a graduate degree as highest completed education. However, there is diversity also in this category; $26 \%$ had a secondary education, and a small percentage (3\%) had primary school as highest completed educational level.

From a modern segmentation perspective, and in comparison with the 2018 baseline study, we found an overrepresentation of participants with the "aware and committed" perspective ( $38 \%$ versus $22 \%$ ) and an underrepresentation of both the "down-to-earth and confident" (11\% versus $20 \%$ ) and the "egalitarian and solidary" perspective ( $28 \%$ versus $37 \%$ ).

\section{CITIZEN SCIENTISTS' ACTIVITIES}

After the recruitment phase, including filling in the presurvey, all registered citizen scientists were invited to attend one of the two "kick-off" meetings, which introduced the project and provided the opportunity to get acquainted with each other and to hand out the water test kit. The water test kit contained a drop test for the determination of hardness (Visocolor HE, Total Hardness H 20 F, Macherey- 
Nagel), a plastic measuring jug, a cooking pot, two pipettes, a tight plunger syringe, and step-by-step instructions.

During the course of the project, participants were invited to take measurements within two different 14-day measurement periods: before and after the installation of the central softener. The time interval between the two phases was planned to be 4 months. During the project realization, however, the COVID-19 pandemic, along with delays in the construction of the new system, led to the second measurement being delayed. In effect, the first measurement period, which started about 10 days after the kick-off meeting, took place in November 2019, whereas the second measurement took place in October 2020. Using a drop test and a simplified boilingwater test, the participants determined the hardness and lime deposition of their water. The drop test is based on a principle of titration by drop counting, and was explained in a step-by-step manner in both an instruction manual and a video. In short, people were asked to fill the test tube and to add 2 drops indicator solution, after which the test sample turns red. Next, people were asked to add titration solution dropwise while smoothly shaking the test tube until the solution turns completely green. At that point, people were asked to read off the total hardness indication (in $\mathrm{mol} / \mathrm{m}^{3}$ ) from the syringe barrel. In each round, the drop test was carried out twice; once on water directly from the tap and once on water that had been boiled for 5 minutes. To this end, people were asked to measure $5 \mathrm{ml}$ of water into their distributed measuring jug and boil this water in the distributed pot without a lid. After boiling, people were asked to cool the water for at least 20 minutes, and again measure the remaining water in the jug. In addition to the second drop test, people were asked to evaluate the quantity of lime deposition in the test pot qualitatively by means of visual assessment. After each measurement round, the citizen scientists were asked to enter their measurement data in an online survey. Figure 2 visualizes some of the activities of the citizen scientists.

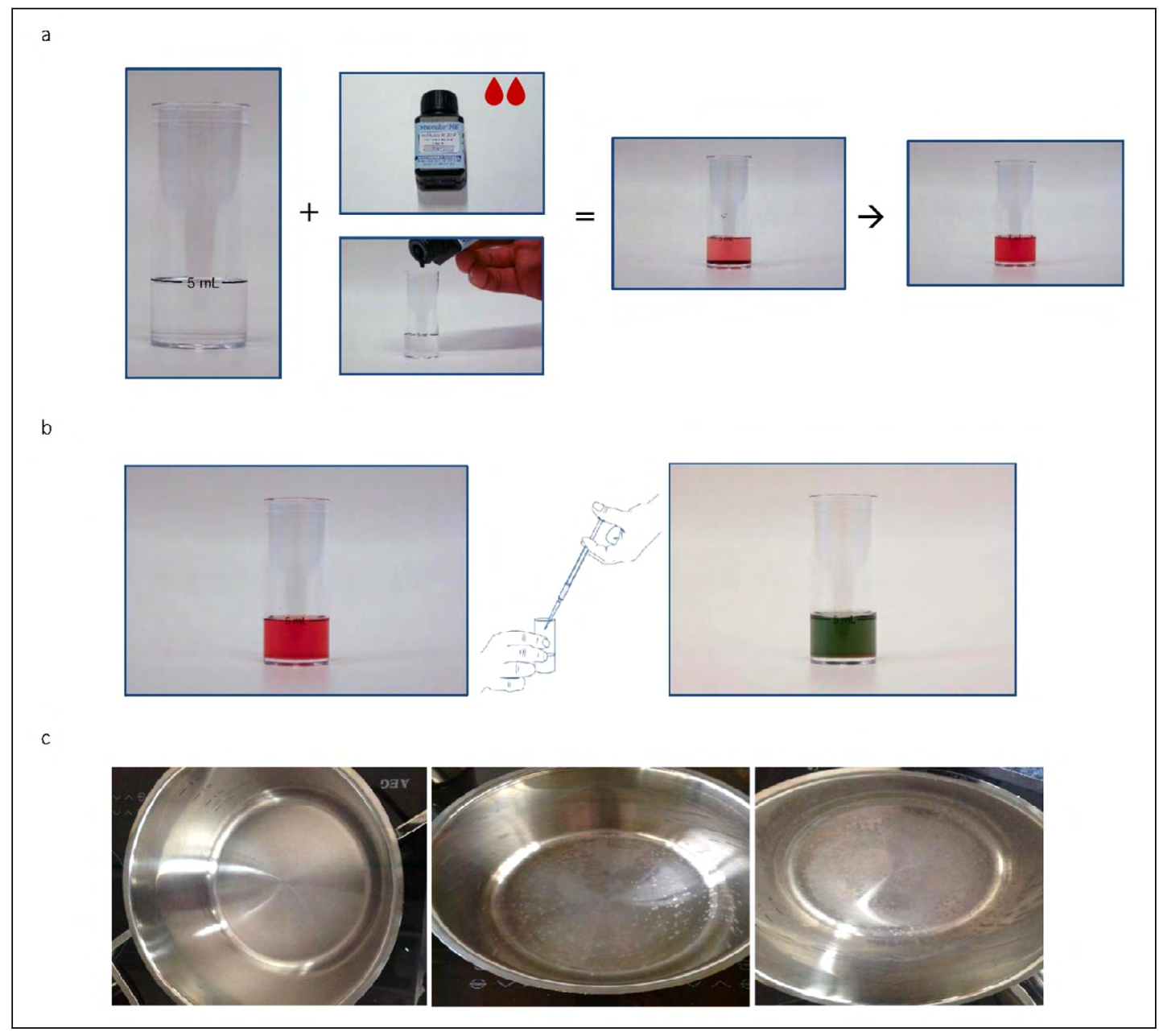

Figure 2 Visualization of different citizen science activities: (a) filling the test tube with $5 \mathrm{ml}$ of tap water with pipette and adding 2 drops indicator solution; (b) using a tight plunger syringe to add titration solution dropwise until the solution turns completely green; and (c) visual assessment of cooking pot. 
Acknowledging the importance of effective communication and feedback in CS (Capdevila et al. 2020), intermediate results were shared via the project website, along with two separate e-mails and a video explaining the analysis, quality control, and interpretation of the measurement results. In addition, CS participants were kept informed about the progress of the project, the reasons for the delay, and subsequent steps. After the analysis of second-round data, the results were shared via a webinar, giving ample opportunities for questions and feedback.

\section{CITIZEN SCIENCE WATER HARDNESS MEASUREMENTS}

In the first measurement round, the total number of participants that shared their data was 130 out of 152 registered participants, representing a participation rate of $86 \%$. In the second round, 98 participants shared their data, representing a $65 \%$ participation rate. In line with the majority of CS studies in the water sciences, to ensure the production of scientifically valid data (Njue et al. 2019), all data points following the CS measurements were subjected to quality control to identify erroneous entries. The following data points were marked as erroneous: (i) registrations with improbably high boiling hardness (measured values of hardness larger than $50^{\circ} \mathrm{fH}$ in the first round, and $70^{\circ} \mathrm{fH}$ in the second round); (ii) values that were reported by citizen scientists themselves as measured "out of protocol"; and (iii) entries with a zero value, before or after boiling. In the first round, $2.3 \%$ of the observations flagged as erroneous required removal; in the second round, this was $7.1 \%$. With a mean error rate for both phases being
$4.7 \%$, this result is considered on par with the error rate seen in sensor measurements for water, which typically assumes measurement confidence intervals of 95\% (Da Silva 2013; EURAMET 2011). The quality of the CS dataset was further validated by a comparison with formal hardness measurements taken by the utility. As further detailed in Supplemental File 2: Data Validation, the validation concludes that the CS measurements have an average error (deviation) rate of $3.56 \%$ when compared with the utility measurements, which is considered on par with confidence intervals typically seen in preliminary sensor measurements (Aceves-Bueno et al. 2017; Sharma et al., 2014) and other studies suggesting that citizen scientists generate high-quality data comparable to professional data (e.g. Storey et al. 2016; Loperfido et al. 2010).

The first thing that becomes apparent from the CS measurements is the effect of the central softener. Figure 3 shows that the average measured hardness of water before boiling dropped from $43.7^{\circ} \mathrm{fH}$ in the first round (which, as listed in Table 1, is classified as hard to very hard) to $20.6^{\circ} \mathrm{fH}$ in the second round (classified as average hard), and from $17.5^{\circ} \mathrm{fH}$ to $11.4^{\circ} \mathrm{fH}$ following the boiling process. It should be noted these after-boiling data are provided after a volume correction for evaporation. This correction is needed because the density of minerals ( $\mathrm{Ca}, \mathrm{Mg}$ ) within boiled water is higher due to evaporation, and entails a recalculation of the decreased volume so as to scale with the original volume of $5 \mathrm{ml}$.

In this study, CS participants not only measured for the hardness of water, but also looked explicitly at the calcium precipitation (PCCP). From the large difference of hardness

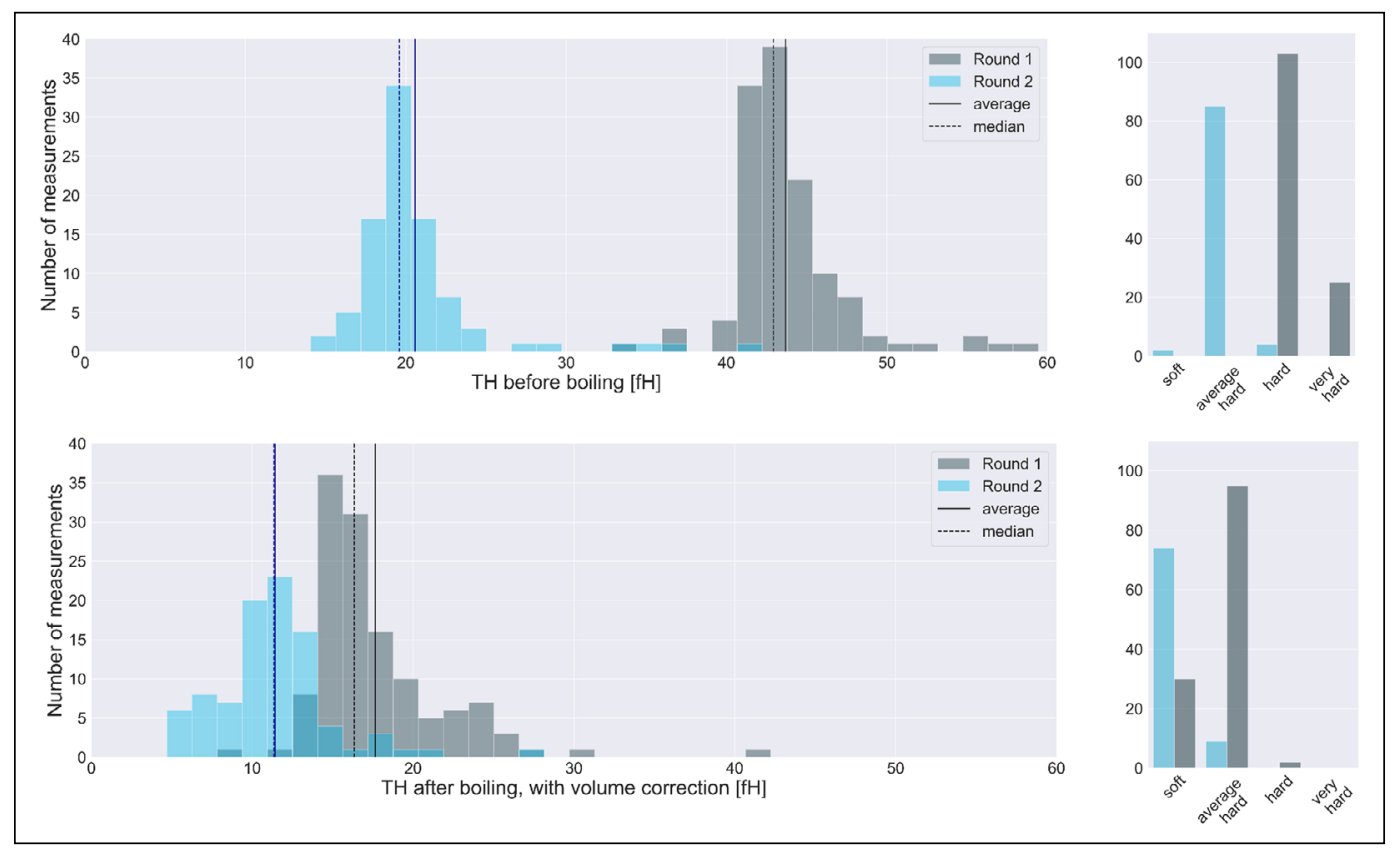

Figure 3 Total hardness (TH) measurements before and after the boiling test. 
before and after boiling in the first round as depicted in Figure 3 , one can deduce a rather high PCCP, whereas in the second round this difference was significantly smaller. As shown in Figure 4, the average PCCP indeed drops from 2.61 $\mathrm{mol} / \mathrm{m}^{3}$ (SD $0.41 \mathrm{~mol} / \mathrm{m}^{3}$ ) in the first round to $0.91 \mathrm{~mol} / \mathrm{m}^{3}$ $\left(\mathrm{SD} 0.40 \mathrm{~mol} / \mathrm{m}^{3}\right)$ in the second round. Accordingly, citizen scientists' measurements indicate a PCCP drop after the installation of the central softeners of $1.69 \mathrm{~mol} / \mathrm{m}^{3}$.

In addition to this quantitative PCCP assessment, by using three sample pictures, participants were asked to qualitatively classify the amount of calcium precipitation in their own cooking pot as "a lot," "limited," or "none" following the boiling test. In accordance with the quantitative data, this classification methodology revealed a significant change. Whereas in the first round, a large majority (88.5\%) classified the inside of their cooking pot as having a lot of calcium precipitation, in the second round, this percentage dropped to $8.2 \%$. Concurrently, the percentage of participants classifying their pot as having a limited calcium precipitation increased from $11.5 \%$ to $86.6 \%$.

\section{RESULTS}

As elaborated, the aim of this paper is to explore how drinking water perceptions and behavior change after the installation of a central softener, and how, if at all, the impact of this intervention differs between water customers directly involved in a connected CS project (the group of citizen scientists) and those not involved in this project (the control group). This section first describes the outcomes related to perceptions and then goes into the results related to behavior change. Detailed information about the results of the statistical tests are provided in Supplemental File 3: Statistical Testing Summary.

\section{PERCEPTION}

With regards to the perceived hardness of water, we observe differences between the pre and post datasets (see Figure 5 for a visual comparison), that are strongly significant for both the CS and control group ( $p<0.001$ in both cases for the Wilcoxon-Mann-Whitney test). For instance, whereas in

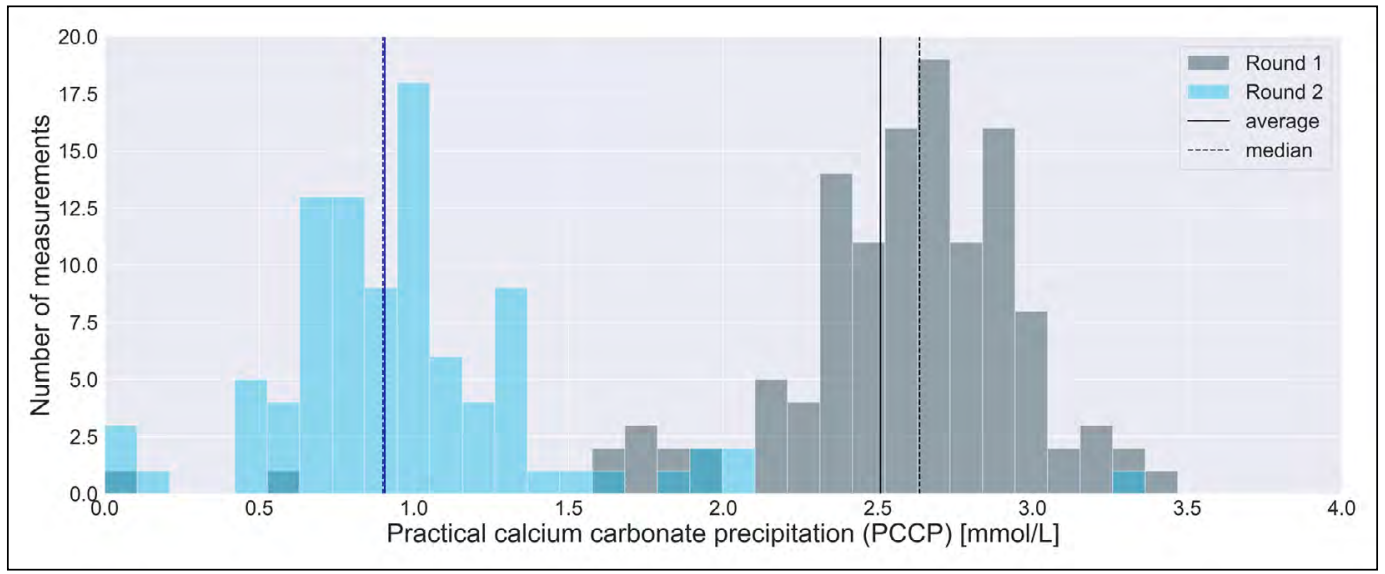

Figure 4 Practical calcium carbonate precipitation (PCCP), derived from hardness measurements.

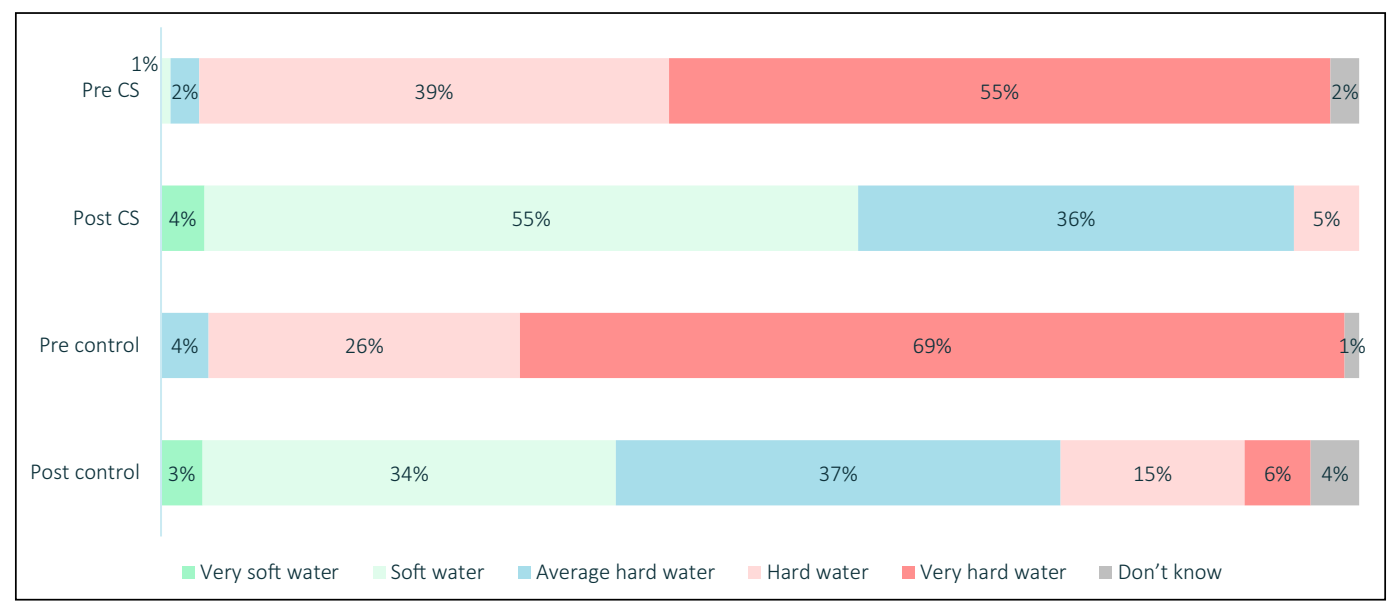

Figure 5 Perceived hardness of water. 
the pre-phase, $55 \%$ of the CS participants and $69 \%$ of the control group perceived their water to be very hard, in the post-phase this was reduced to respectively $0 \%$ and $6 \%$. Likewise, we find that the percentage of respondents who regard their water as soft grew from $1 \%$ and $0 \%$ to $39 \%$ and $26 \%$. The shift in perception appears to be stronger among the CS participants, both in the pre- $(p=0.043$, Wilcoxon-Mann-Whitney test) and the post-phase ( $p=$ 0.018 , Wilcoxon-Mann-Whitney test).

It is noteworthy that after the installation of the central softener, customers not only perceive their water as softer, but also as more healthy, and to a lesser degree, more tasteful ( $p<0.001$ between the pre- and post-phases in both datasets, Wilcoxon-Mann-Whitney test). For instance, the percentage of CS participants perceiving their water to be healthy grows from $58 \%$ to $86 \%$ and perceiving their water as having a good taste from $52 \%$ to $79 \%$. These differences are summarized in Table 3. Interestingly, the CS participants seem to magnify these perception differences even more against the control datasets $(p<$ 0.05 during the pre-phase, $p<0.1$ during the post-phase). The lower statistical significance in this magnification can be attributed to the small number of CS participants, particularly in the post-phase. Accordingly, we observe strong reductions in the concerns about the quality of tap water between the two phases $(p<0.001$ in both the control and CS group comparisons, Wilcoxon-MannWhitney test), which are magnified more between the CS and control group in the post-phase $(p<0.001$, WilcoxonMann-Whitney test) but interestingly not in the pre-phase $(p>1$, Wilcoxon-Mann-Whitney test). We also observe growing concerns for rising prices ( $p<0.001$ for both groups, accordingly, Wilcoxon-Mann-Whitney test), which are also more profound in the CS participants compared with the control group, especially in the post-phase $(p=0.057$ and $p$ $=0.002$ when comparing pre and post phases, accordingly, Wilcoxon-Mann-Whitney). This growing concern, however, cannot simply be attributed to the softener, as the study period coincided with the circumstances associated with the COVID-19 pandemic that led to major concerns about job security and concerns about how substantially more time indoors translates into higher utility bills.

The same pattern can be seen when looking at the calcium precipitation inconvenience that customers experience. Table 4 shows strong differences between the pre- and post-phase across all datasets $(p<0.001$ for both warm and cold water, for both groups, Wilcoxon-Mann-Whitney test). Again, a stronger shift in perception among the CS participants is observed, especially in relation to the use of cold water $(p=0.02-0.05$ and $p<0.001$ when comparing control and CS groups for both phases and for warm and cold water respectively, Wilcoxon-Mann-Whitney test).

\section{Appreciation scores}

As shown in Table 5, the strong perception shifts after the softening of water also result in changes in the average

\begin{tabular}{|c|c|c|c|c|c|c|c|c|c|c|}
\hline & \multicolumn{10}{|c|}{ CITIZEN SCIENCE PARTICIPANTS } \\
\hline & \multicolumn{5}{|l|}{ PRE } & \multicolumn{5}{|l|}{ POST } \\
\hline & -- & - & o & + & ++ & -- & - & o & + & ++ \\
\hline Healthy & $0 \%$ & $4 \%$ & $30 \%$ & $47 \%$ & $11 \%$ & $0 \%$ & $0 \%$ & $13 \%$ & $43 \%$ & $43 \%$ \\
\hline Good taste & $2 \%$ & $11 \%$ & $33 \%$ & $42 \%$ & $10 \%$ & $0 \%$ & $2 \%$ & $2 \%$ & $48 \%$ & $31 \%$ \\
\hline Quality concerns & $6 \%$ & $41 \%$ & - & $42 \%$ & $11 \%$ & $33 \%$ & $58 \%$ & - & $5 \%$ & $4 \%$ \\
\hline \multirow[t]{4}{*}{ Price increase concerns } & $3 \%$ & $16 \%$ & - & $44 \%$ & $36 \%$ & $9 \%$ & $25 \%$ & - & $49 \%$ & $16 \%$ \\
\hline & \multicolumn{10}{|c|}{ CONTROL GROUP } \\
\hline & \multicolumn{5}{|l|}{ PRE } & \multicolumn{5}{|l|}{ POST } \\
\hline & -- & - & o & + & ++ & -- & - & o & + & ++ \\
\hline Healthy & $4 \%$ & $5 \%$ & $37 \%$ & $31 \%$ & $12 \%$ & $1 \%$ & $3 \%$ & $25 \%$ & $37 \%$ & $25 \%$ \\
\hline Good taste & $7 \%$ & $14 \%$ & $36 \%$ & $25 \%$ & $13 \%$ & $2 \%$ & $5 \%$ & $29 \%$ & $36 \%$ & $22 \%$ \\
\hline Quality concerns & $6 \%$ & $39 \%$ & - & $38 \%$ & $17 \%$ & $21 \%$ & $50 \%$ & - & $21 \%$ & $6 \%$ \\
\hline Price increase concerns & $2 \%$ & $10 \%$ & - & $43 \%$ & $44 \%$ & $5 \%$ & $15 \%$ & - & $44 \%$ & $35 \%$ \\
\hline
\end{tabular}

Table 3 Perceptions about drinking water, expressed in the range of disagreement/agreement (five possible responses) for health and good taste) and little or many concerns (four possible responses) for the water quality and price increase.

Notes: Pre control, $n=435$; Pre CS, $n=142$; Post control, $n=497$; Post CS, $n=61$. Quality and price-concern data are exclusive of customers who recently experienced a malfunction. 


\begin{tabular}{|c|c|c|c|c|c|}
\hline & \multirow[b]{2}{*}{ INCONVENIENCE } & \multicolumn{2}{|c|}{$\begin{array}{l}\text { CITIZEN SCIENCE } \\
\text { PARTICIPANTS }\end{array}$} & \multicolumn{2}{|c|}{ CONTROL GROUP } \\
\hline & & PRE & POST & PRE & POST \\
\hline \multirow[t]{4}{*}{ Cold water } & No & $7 \%$ & $64 \%$ & $7 \%$ & $39 \%$ \\
\hline & A little & $50 \%$ & $35 \%$ & $31 \%$ & $50 \%$ \\
\hline & Quite a bit & $25 \%$ & $2 \%$ & $35 \%$ & $7 \%$ \\
\hline & Very much & $18 \%$ & $0 \%$ & $28 \%$ & $4 \%$ \\
\hline \multirow[t]{4}{*}{ Warm water } & No & $1 \%$ & $18 \%$ & $2 \%$ & $15 \%$ \\
\hline & A little & $17 \%$ & $78 \%$ & $17 \%$ & $65 \%$ \\
\hline & Quite a bit & $42 \%$ & $4 \%$ & $32 \%$ & $14 \%$ \\
\hline & Very much & $40 \%$ & $0 \%$ & $50 \%$ & $6 \%$ \\
\hline
\end{tabular}

Table 4 Experienced inconvenience due to calcium precipitation.

Notes: Pre control, $n=327$; pre $C S, n=125$; post control, $n=345$; post CS, $n=55$; data are exclusive of customers with a personal water softener).

customer appreciation scores for water quality, the price/quality ratio, and the confidence in the utility. Indeed, our data show a strongly significant difference between the appreciation score for quality before and after the installation of the central water softener $(p<0.001$ in both cases, control and CS, for the Wilcoxon-Mann-Whitney test, $p<0.001$ for chi-squared test in the control dataset). We also note significant differences in the average water quality score between the control and CS groups before and after the installation (when compared in pairs), for both the pre- and the post-phase ( $p<0.001$ for the Wilcoxon-MannWhitney test).

Regarding confidence in the utility, the data shows a significantly higher score following the softener installation ( $p<0.001$ in both groups for the Mann-Whitney $U$ test), which is even more profound among the CS participants when compared with the control group $(p<0.001$ in both cases for the Mann-Whitney $U$ test). Likewise, we observe the same effects in the price/quality ratio scoring $(p<0.001$ in all cases, both tests). In conclusion, it appears that the softening of water not only boosts the perceived quality of drinking water and price/quality ratio, but also the confidence in the drinking water utility; on all occasions, these differences are more profound in the CS datasets. For instance, Table 5 shows that in the post-phase, CS participants gave appreciation scores that are on average $10 \%$ higher than the control group (in all three perception rankings).

In addition to the analyses between pre and post and CS versus control group, we assessed whether we could observe strong differences between different types of customers. Shifts in appreciation scores between traditional

\begin{tabular}{lllllll}
\hline & \multicolumn{2}{l}{$\begin{array}{l}\text { CITIZEN SCIENCE } \\
\text { PARTICIPANTS }\end{array}$} & & \multicolumn{2}{l}{$\begin{array}{l}\text { CONTROL } \\
\text { GROUP }\end{array}$} \\
\cline { 2 - 3 } \cline { 5 - 6 } \cline { 5 - 6 } & PRE & POST & & PRE & POST \\
\hline Quality water & 6.6 & 8.6 & & 5.8 & 8 \\
\hline $\begin{array}{lllllll}\text { Price/quality ratio of } \\
\text { water }\end{array}$ & 6.3 & 7.6 & & 5.4 & 6.8 \\
\hline Confidence in utility & 7.6 & 8.7 & & 7.1 & 7.9 \\
\hline
\end{tabular}

Table 5 Average appreciation scores on a scale from 1 to 10. Notes: Pre control, $n=435$; pre CS, $n=142$; post control, $n=497$; post $C S, n=61$. Data are exclusive of customers who recently experienced a malfunction.

socio-demographic variables (gender, age, education) were largely not or hardly distinctive. In coherence with Koop et al. (2021), segmentation based on drinking water customer perspectives instead show moderate differences among profiles in the utility confidence scores and-to a lesser degree-to the price/quality ratio. However, we cannot deduce reliable results for CS participants from this segmentation analysis, mainly owing to the small dataset of post-CS participants that leads to sparse segmented contingency tables. Further details of segmentation data are provided in Supplemental File 4: Segmentation Data.

\section{Perceived hardness vis-à-vis quality}

As mentioned, a strong correlation between the perception of tap water quality and the perception of hardness was observed in the 2018 baseline study. We also observe this in our study for both the control group and for CS participants, as seen in Figure 6, with the vertical lines depicting the $95 \%$ confidence intervals of scoring. Interestingly, CS 


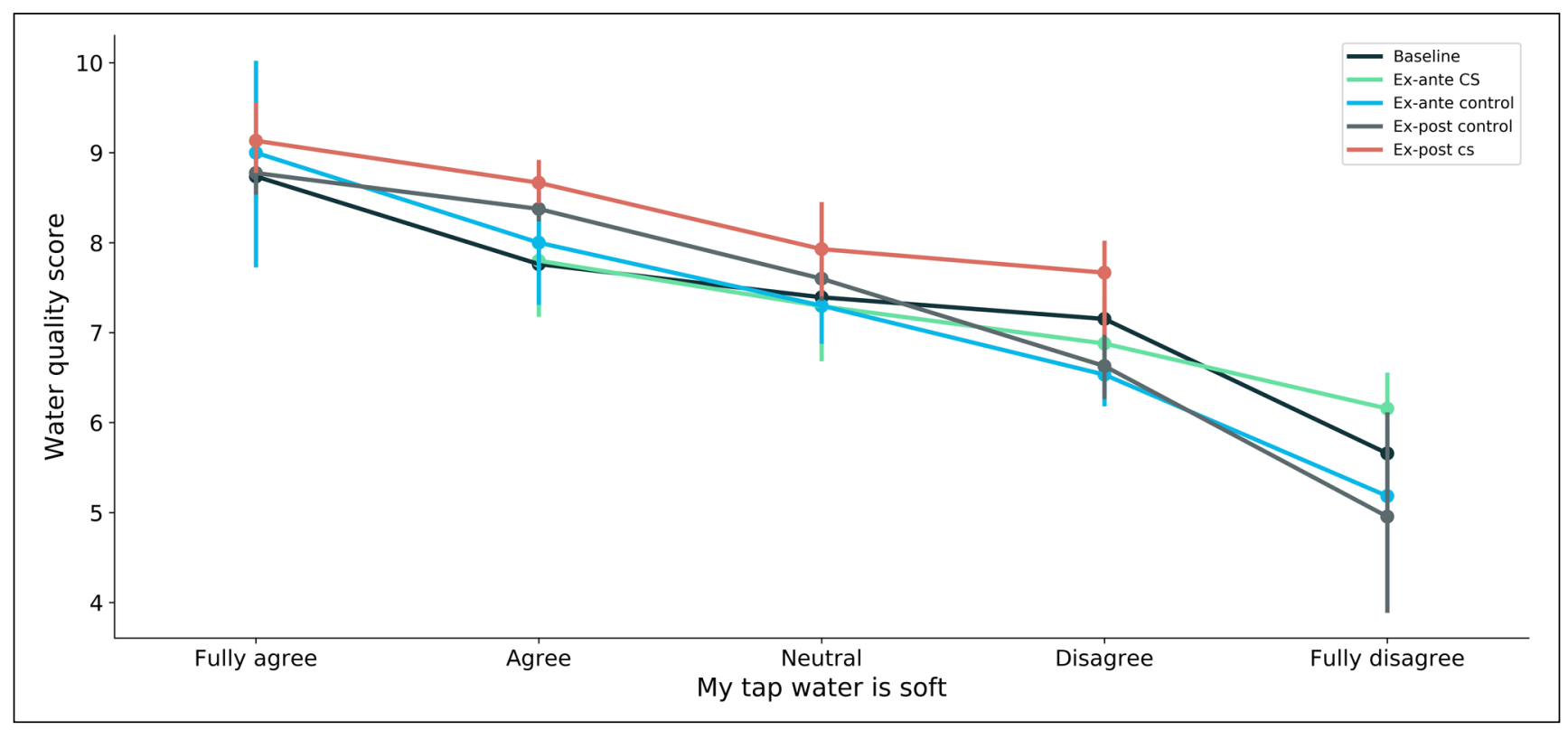

Figure 6 Correlation between the perceived hardness and the quality of water.

\begin{tabular}{llllll}
\hline $\begin{array}{l}\text { MEAN FREQUENCY } \bar{f}_{i} \\
\text { [TIMES/PERSON/YEAR] }\end{array}$ & $\begin{array}{l}\text { WASHING } \\
\text { MACHINE }\end{array}$ & $\begin{array}{l}\text { ELECTRIC } \\
\text { KETTLE }\end{array}$ & $\begin{array}{l}\text { COFFEE } \\
\text { MACHINE }\end{array}$ & DISHWASHER \\
\hline Citizen science participants & Pre & 14.2 & 11.8 & 15.9 & 184.9 \\
\cline { 2 - 6 } & Post & 4.4 & 6.4 & 9.6 & 104.2 \\
\hline Control group & Pre & 13.1 & 14.0 & 18.6 & 162.3 \\
\cline { 2 - 6 } & Post & 8.1 & 10.0 & 12.8 & 136.7 \\
\hline
\end{tabular}

Table 6 Average frequency of use of calcium-removal products.

participants tended to give higher water quality scores regardless of their hardness perception, and this effect became more profound in the post-phase.

\section{BEHAVIOR}

To elucidate to what extent, if at all, CS participation after the introduction of the softener has resulted in behavior change, we analyzed both the installation of individual water softener systems and the use of calcium-removal products. With the former, we observed a limited reduction. In the spring of $2019,13 \%$ of the CS participants and $26 \%$ of customers within the control group had a softener installed in their household. In December 2020, respectively, 11\% and $13 \%$ disconnected this softener. Furthermore, we found that a large majority had adjusted their personal softener (33\% versus $26 \%$ ) or were planning to do so $(56 \%$ versus $40 \%)$.

Concerning the use of calcium removal products, we examined the qualitative responses related to the frequency of use on a series of household appliances, and thereupon estimated the number of uses per year $f_{\mathrm{i}}$ for each category $i$. On the basis of these assumptions, we then calculated the mean frequency (weighted against the number of responses) $\bar{f}_{i}$, which represents the average uses of calcium removal products per person per year for a particular appliance.

As shown in Table 6, there is a clear frequency reduction of calcium-removal products among all devices following the installation of the softener in both groups (control and (S). This reduction is, however, significantly more pronounced among CS participants.

\section{DISCUSSION AND CONCLUSION}

In this study, the focus was on obtaining a better understanding of the effects on perception and behavior as a result of CS participation, an element generally understudied in the CS literature, both within and beyond the water domain (Jordan et al. 2011; Walker, Smigaj, and Tani 2021). This focus on individual outcomes does not mean, however, that we should lose sight of the system 
and scientific outcomes. Indeed, following Shirk et al. (2012), we take the view that the most valuable CS projects yield scientific, system, and individual outcomes.

Regarding the scientific outcomes of this study, in addition to the general increased understanding of the value and significance of citizen science in the drinking water sector, the Waarmaarde CS project generated valuable data, particularly in relation to lime deposition at the household level and the qualitative observations and questions about individual perceptions that accompanied the quantitative measurements. In this context, and in coherence with Aceves-Bueno et al. (2017), we have seen that the CS measurements at the household level have been reliable and showed an error rate on par with the one considered for more formal sensors.

From a system outcome point of view, and in coherence with previous studies in the drinking water domain (Brouwer and Hessels 2019), the most important conclusion is that this CS project has resulted in a growing confidence in the water utility. In addition, we found that the softening of water can lead to significant positive shifts in the perception of a number of factors, including the hardness, quality, and taste of water. Furthermore, and independently from the CS component, this study established a strong correlation between the perceived and actual hardness of water, and between the perception of hardness and the perceived tap-water quality. The latter suggests that the softening of water offers a strong potential for utilities to boost customers satisfaction and confidence scores. For future research, it would be of interest to assess how these dynamics function in areas where the hardness of water is low, but satisfaction scores fall short.

Finally, and central to this study, from an individualoutcome perspective, we have seen that the installation of a central softener results in both perception and behavior change. Through the design of this study, which not only considered CS participants, but also employed a control group, we observed that shifts in perception of different factors were consistently more profound for the CS participants in aspects such as appreciation, perception of qualitative factors, and concerns about limescale deposits. Moreover, CS participants have shown a higher confidence in the water utility and have given higher appreciation scores about their water quality. The same pattern is observed with respect to how these perception shifts translate into behavioral ones, for instance through the drastic reduction in use of calcium-removal products.

The above conclusions are encouraging for CS research but have certain limitations, which point to further research needs. First, the number of CS participants in this study was relatively limited, especially in the post-phase; for increased statistical validity, future research should encompass a larger set of participants $(n>100)$. A second limitation of this study relates to the method of creating the control group. In the current study, at least part of the drinking water customers belonging to the control group was not willing to participate in the citizen science project. Follow-up research would ideally consist of two groups that are as similar as possible in size, intentions, and attitude. Third, it would be interesting for future research to draw on an extended research design that includes cross-domain and/or international comparisons so as to explore cultural aspects of consumer behavior.

\section{SUPPLEMENTARY FILES}

The supplementary files for this article can be found as follows:

- Supplemental File 1. Survey Questions. DOI: https://doi. org/10.5334/cstp.452.s1

- Supplemental File 2. Data Validation. DOI: https://doi. org/10.5334/cstp.452.s2

- Supplemental File 3. Statistical Testing Summary. DOI: https://doi.org/10.5334/cstp.452.s3

- Supplemental File 4. Segmentation Data. DOI: https:// doi.org/10.5334/cstp.452.s4

\section{ETHICS AND CONSENT}

This study was reviewed and approved by the Chairman of the Scientific Council of KWR Water Research Institute, Prof. Kees van Leeuwen.

\section{ACKNOWLEDGEMENTS}

The authors owe a special debt of gratitude to De Watergroep colleagues Katleen De Leu and Dirk Bloemen and all fellow members of CS project group, and all respondents and participating citizen scientists.

\section{AUTHOR CONTRIBUTIONS}

Stijn Brouwer designed the project and survey methodologies and led the writing; Dimitrios Bouziotas analyzed the data, drafted the corresponding sections of the manuscript, and critically revised other sections. All authors gave final approval of the submitted version and agree to be accountable for aspects of the work they conducted. 


\section{AUTHOR AFFILIATIONS}

Stijn Brouwer (D) orcid.org/0000-0003-1441-2930

KWR Water Research Institute, NL

Department of Sociology, University of Antwerp, Antwerp, Belgium

Dimitrios Bouziotas (D) orcid.org/0000-0003-2172-350X

KWR Water Research Institute, NL

\section{REFERENCES}

Aceves-Bueno, E, Adeleye, AS, Feraud, M, Huang, Y, Tao, M, Yang, $\mathbf{Y}$ and Anderson, SE. 2017. The accuracy of citizen science data: a quantitative review. The Bulletin of the Ecological Society of America, 98: 278-290. DOI: https://doi. org/10.1002/bes2.1336

Ahn, MK, Chilakala, R, Han, C and Thenepalli, T. 2018. Removal of hardness from water samples by a carbonation process with a closed pressure reactor. Water, 10(54). DOI: https:// doi.org/10.3390/w10010054

Babich, R, Craig, E, Muscat, A, Disney, J, Farrell, A, Silka, L and Jayasundara, N. 2021. Defining drinking water metal contaminant mixture risk by coupling zebrafish behavioral analysis with citizen science. Scienfic Reports, 11: 17303. DOI: https://doi.org/10.1038/s41598-021-96244-4

Brouwer, S and Hessels, LK. 2019. Increasing research impact with citizen science: the influence of recruitment strategies on sample diversity. Public Understanding of Science, 28: 606-621. DOI: https://doi.org/10.1177/0963662519840934

Brouwer, S and Sjerps, R. 2018. Klantperspectieven, Nieuwegein, BTO 2018.083, KWR.

Brouwer, S, Pieron, M, Sjerps, R and Etty, T. 2019. Perspectives beyond the meter: A Q-study for modern segmentation of drinking water customers. Water Policy, 21: 1224-1238. DOI: https://doi.org/10.2166/wp.2019.078

Brouwer, S, Van Der Wielen, PWJJ, Schriks, M, Claassen, M and Frijns, J. 2018. Public participation in science: The future and value of citizen science in the drinking water research. Water, 10: 284. DOI: https://doi.org/10.3390/w10030284

Capdevila, ASL, Kokimova, A, Ray, SS, Avellán, T, Kim, J and Kirschke, S. 2020. Success factors for citizen science projects in water quality monitoring. Science of the Total Environment, 728: 137843. DOI: https://doi.org/10.1016/j.scitotenv.2020.137843

Church, SP, Payne, LB, Peel, S and Prokopy, LS. 2019. Beyond water data: benefits to volunteers and to local water from a citizen science program. Journal of Environmental Planning and Management, 62: 306-326. DOI: https://doi.org/10.1080/ 09640568.2017.1415869

D'Alessio, M, Rushing, G and Gray, TL. 2021. Monitoring water quality through citizen science while teaching STEM undergraduate courses during a global pandemic. Science of The Total Environment, 779: 146547. DOI: https://doi. org/10.1016/j.scitotenv.2021.146547
Da Silva, RJNB. 2013. Setting Target Measurement Uncertainty in Water Analysis. Water, 5: 1279-1302. DOI: https://doi. org/10.3390/w5031279

Egerer, MH, Lin, BB and Philpott, SM. 2018. Water use behavior, learning, and adaptation to future change in urban gardens. Frontiers in Sustainable Food Systems, 2: 71. DOI: https://doi. org/10.3389/fsufs.2018.00071

Euramet. 2011. Guidelines on the Estimation of Uncertainty in Hardness Measurements. Version 2.0 (03/2011) ed. Braunschweig: EURAMET e.V.

Ferri, M, Wehn, U, See, L, Monego, M and Fritz, S. 2020. The value of citizen science for flood risk reduction: cost-benefit analysis of a citizen observatory in the Brenta-Bacchiglione catchment. Hydrology and Earth System Sciences, 24: 57815798. DOI: https://doi.org/10.5194/hess-24-5781-2020

Groenendijk, M, Van De Wetering, $\mathbf{S}$ and Van Nieuwenhuijze, $\mathbf{R}$. 2008. Central water softening: Customer comfort is relevant in new WHO view. Water Science and Technology: Water Supply, 8: 69-74. DOI: https://doi.org/10.2166/ws.2008.036

Harriden, K. 2013. Water Diaries: generate intra-household water use data - generate water use behaviour change. Journal of Water, Sanitation and Hygiene for Development, 3: 70-80. DOI: https://doi.org/10.2166/washdev.2013.015

Haywood, BK. 2016. Beyond data points and research contributions: the personal meaning and value associated with public participation in scientific research. International Journal of Science Education, Part B, 6: 239-262. DOI: https:// doi.org/10.1080/21548455.2015.1043659

Irwin, A. 1995. Citizen science: A study of people, expertise and sustainable development, London, New-York, Routledge. DOI: https://doi.org/10.4324/9780203202395

Jakositz, S, Pillsbury, L, Greenwood, S, Fahnestock, M, Mcgreavy, B, Bryce, J and Mo, W. 2020. Protection through participation: Crowdsourced tap water quality monitoring for enhanced public health. Water research, 169: 115209. DOI: https://doi.org/10.1016/j.watres.2019.115209

Jordan, RC, Gray, SA, Howe, DV, Brooks, WR and Ehrenfeld, JG. 2011. Knowledge gain and behavioral change in citizenscience programs. Conservation biology, 25: 1148-1154. DOI: https://doi.org/10.1111/j.1523-1739.2011.01745.x

Koop, SHA, Clevers, SHP, Blokker, EJM and Brouwer, S. 2021. Public Attitudes towards Digital Water Meters for Households. Sustainability, 13: 6440. DOI: https://doi.org/10.3390/ su13116440

Land-Zandstra, A, Agnello, G and Gültekin, YS. 2021. Participants in Citizen Science. In: Vohland, K, Land-Zandstra, A, Ceccaroni, L, Lemmens, R, Perelló, J, Ponti, M, Samson, R and Wagenknecht, K. (eds.), The Science of Citizen Science. Cham, Switzerland: Springer. DOI: https://doi.org/10.1007/978-3030-58278-4

Lanz, B and Provins, A. 2016. The demand for tap water quality: Survey evidence on water hardness and aesthetic quality. 
Water Resources and Economics, 16: 52-63. DOI: https://doi. org/10.1016/j.wre.2016.10.001

Li, $\mathbf{P}$ and $\mathbf{W u}, \mathbf{J} .2019$. Drinking water quality and public health. Exposure and Health, 11: 73-79. DOI: https://doi.org/10.1007/ s12403-019-00299-8

Loperfido, JV, Beyer, P, Just, CL and Schnoor, JL. 2010. Uses and biases of volunteer water quality data. Environmental Science \& Technology, 44: 7193-7199. DOI: https://doi.org/10.1021/ es100164C

Njue, N, Kroese, JS, Gräf, J, Jacobs, S, Weeser, B, Breuer, $\mathbf{L}$ and Rufino, M. 2019. Citizen science in hydrological monitoring and ecosystem services management: State of the art and future prospects. Science of the Total Environment, 693: 133531. DOI: https://doi.org/10.1016/j. scitotenv.2019.07.337

Odetola, L, Sills, S and Morrison, S. 2021. A pilot study on the feasibility of testing residential tap water in North Carolina: Implications for environmental justice and health. Journal of Exposure Science \& Environmental Epidemiology. DOI: https:// doi.org/10.1038/s41370-021-00352-2

Pudifoot, B, Cárdenas, ML, Buytaert, W, Paul, JD, Narraway, CL and Loiselle, S. 2021. When it rains, it pours: Integrating citizen science methods to understand resilience of urban green spaces. Frontiers in Water, 3: 654493. DOI: https://doi. org/10.3389/frwa.2021.654493

Redmon, JH, Levine, KE, Aceituno, AM, Litzenberger, $\mathrm{K}$ and Gibson, JM. 2020. Lead in drinking water at North Carolina childcare centers: Piloting a citizen science-based testing strategy. Environmental research, 183: 109126. DOI: https:// doi.org/10.1016/j.envres.2020.109126

Roy, S and Edwards, M. 2019. Citizen Science During the Flint, Michigan Federal Water Emergency: Ethical Dilemmas and
Lessons Learned. Citizen Science: Theory and Practice, 4: 12.

DOI: http://doi.org/10.5334/cstp.154

Sharma, M, Sharma, R and McBean, EA. 2014. Enhancing confidence in drinking water quality for improved risk decisions. Human and Ecological Risk Assessment: An International Journal, 20(5): 1281-1290. DOI: https://doi.org/ 10.1080/10807039.2013.828514

Shirk, JL, Ballard, HL, Wilderman, CC, Phillips, T, Wiggins, A, Jordan, R, Mccallie, E, Minarchek, M, Lewenstein, BV and Krasny, ME. 2012. Public participation in scientific research: a framework for deliberate design. Ecology and Society, 17: 29 DOI: https://doi.org/10.5751/ES-04705-170229

Stepenuck, KF and Green, LT. 2015. Individual-and communitylevel impacts of volunteer environmental monitoring: a synthesis of peer-reviewed literature. Ecology and society, 20: 19. DOI: https://doi.org/10.5751/ES-07329-200319

Storey, RG, Wright-Stow, A, Kin, E, Davies-Colley, RJ and Stott, R. 2016. Volunteer stream monitoring: Do the data quality and monitoring experience support increased community involvement in freshwater decision making? Ecology and Society, 21: 32. DOI: https://doi.org/10.5751/ES-08934210432

Topping, M and Kolok, A. 2021. Assessing the accuracy of nitrate concentration data for water quality monitoring using visual and cell phone quantification methods. Citizen Science: Theory and Practice, 6: 1, 5. DOI: https://doi.org/10.5334/ cstp.346

Walker, DW, Smigaj, M and Tani, M. 2021. The benefits and negative impacts of citizen science applications to water as experienced by participants and communities. Wiley Interdisciplinary Reviews: Water, 8: e1488. DOI: https://doi. org/10.1002/wat2.1488

\section{TO CITE THIS ARTICLE:}

Brouwer, S and Bouziotas, D. 2022. Water Hardness in the Eye of the Beholder: Exploring Links between Central Softening, Customer Perception and Behavior, and Citizen Science. Citizen Science: Theory and Practice, 7(1): 2, pp. 1-14. DOI: https://doi.org/10.5334/cstp.452

Submitted: 01 July 2021 Accepted: 08 January 2022 Published: 24 January 2022

\section{COPYRIGHT:}

(c) 2022 The Author(s). This is an open-access article distributed under the terms of the Creative Commons Attribution 4.0 International License (CC-BY 4.0), which permits unrestricted use, distribution, and reproduction in any medium, provided the original author and source are credited. See http://creativecommons.org/licenses/by/4.0/.

Citizen Science: Theory and Practice is a peer-reviewed open access journal published by Ubiquity Press.

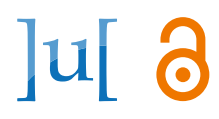

